\title{
NUMERICAL INVESTIGATION OF DIFFERENT RANS MODEL ON SUPERSONIC JET IN PRESENCE OF PASSIVE JET CONTROLS
}

\author{
DEVENDRA KR. LOHIA ${ }^{1}$, DR. H.K. PALIWAL ${ }^{2} \&$ DR. SHANTANU SRIVASTAVA ${ }^{3}$ \\ ${ }^{1}$ Department of Mechanical Engineering, Faculty of Engineering and Technology, Rama University,
}

Kanpur, Uttar Pradesh, India

${ }^{2}$ Department of Mechanical Engineering, Institute of Engineering and Technology, Lucknow, Uttar Pradesh, India

${ }^{3}$ Department of Mechanical Engineering, MNNIT, Allahabad Prayagraj, Allahabad, Uttar Pradesh, India

\begin{abstract}
A nozzle is equipped for changing, transforming pressure energy into kinetic energy and the other way around, depending on the state of the nozzle above changes happen. Nozzle which is a C-D nozzle is utilized to achieve supersonic stream speeds. The inlet Mach number is less than one; the convergent section speeds up it to the sonic speed at the throat and further sped up to supersonic speeds by the diverging section. A nozzle is a device intended to control the direction or attributes of a fluid flow (particularly to expand speed) as it exits (or enters) an encased pipe. A nozzle is frequently a channel or container of shifting cross-sectional zone and it very well may coordinate or change the progression of a liquid (gas). Nozzles are as often as possible used to control the speed of stream, speed, and direction, mass, shape, and/or the pressure of the stream that emerges from them. To improve the mixing attributes execution of supersonic controlled stream using limiting tab, which is the dainty wire that makes $5 \%$ obstruction of the diameter across of the nozzle exit. Different Reynolds Averaged Navier Strokes Equation RANS models are using and look at the consequences of Mach no. 1.86 supersonic stream from 2D Convergent-Divergent nozzles for NPR 4 with a step size of one, to examine diverse scientific models for execution. Converging the over-expanded and close to addressing development condition ANSYS Fluent 16.0. Using 2D hex components and organized work has produced into the ICEM module. Non-dimensional plots of Mach No along fly focus line, regarding the non-dimensional separation of $k-\varepsilon$ workable, $k-\omega, k$ - $\omega$ standard, Reynolds Stress with standard and change SST conditions are looked at and investigations.
\end{abstract}

KEYWORDS: RANS Model, Jet Mixing, Centerline Pressure Decay, Mach Number, NPR, Passive Controls

Received: Jun 08, 2020; Accepted: Jun 28, 2020; Published: Sep 17, 2020; Paper Id.: IJMPERDJUN20201308

\section{INTRODUCTION}

The mixing characteristic is a very important area in the jets there is a verity of technique is used for improving the mixing characteristic Enhancement of supersonic jet mixing important in several applications includes noise reduction in the jet exhaust and improvement of flow distribution within the acoustic wind tunnel. A foremost finish of this examination is that the pivotal vortices blending instrument was before showed to be answerable for low-speed subsonic

streams fast mixing are powerful. It is likewise successful in a supersonic stream condition. The control technique is used to enhancing the mixing in the jets. In the passive controls, limiting tabs are used on the nozzle exit. It generates the vortex through the tab to improve mixing and decrease the core length of the plume. In 
the present study rectangular cross-section is taken for the tab overall it develops the $5 \%$ resistance in nozzle exit the application of a reliable method in computing the supersonic jet flow field description originated in the 90s. The Reynolds-Averaged Navier-Stokes (RANS) [1] system is the most predictable come close to estimate the shear flows. The RANS technique consists of time-averaged at the conditions of movement for fluid stream. Reynolds projected the method of Reynolds decomposition, where a direct quantity, for example, ' $u$ ' is decomposed into $\bar{u}$ time-averaged and random quantities correspondingly. The RANS [2] conditions are essentially utilized in dissecting turbulent flows, with guess dependent on information on the stream properties to give assessed time-averaged answers for the Navier-Stokes conditions. In this paper attempts various RANS turbulence model for a vast mainstream of calculations. Two-equation models, such as k- $\varepsilon$, SST formulations, and k- $\omega$ model are widely used to solve for the turbulent kinetic energy in the jet environment.

\subsection{Types of Jet Control}

Two techniques [3] to be specific, dynamic control and inactive control are utilized for stream control. In the dynamic control technique, an extra wellspring of energy is required to actuate the control. Though, in the inactive control systems, alteration of spout leave geometry or presenting little projection as tab is utilized as the control gadgets, without the requirement for an extra wellspring of energy.

\subsubsection{Active Control of Jet}

Numerous active jet control strategies use empowered actuators to progressively control stream wonders dependent on open-or shut circle calculations. Beat planes, piezoelectric actuators, and oscillating jets are among the best controls for dynamic blending improvement. The design of an active flow control system requires an understanding of flow fact and choice of proper actuators, sensors, and a control algorithm. The role of an actuator is to inject perturbations at a prescribed frequency into the flow at locations where the flow is most accessible to these inputs. actuator leverages or disrupts the flow to bring about the desired effect.

\subsubsection{Passive Control of Jet}

The passive control Figure (a.) method are used mainly to create volatility in the shear layer by disturbing the boundary layer at the nozzle exit in the form of grooves[4] along the circumference at the nozzle exit, a tab of different configurations namely rectangular and triangular. No outer force is required in this strategy. The tabs placed at the nozzle exit create streamwise vortices which increase the [5] mixing. Tabs are built to resist the high temperature by choosing proper material.

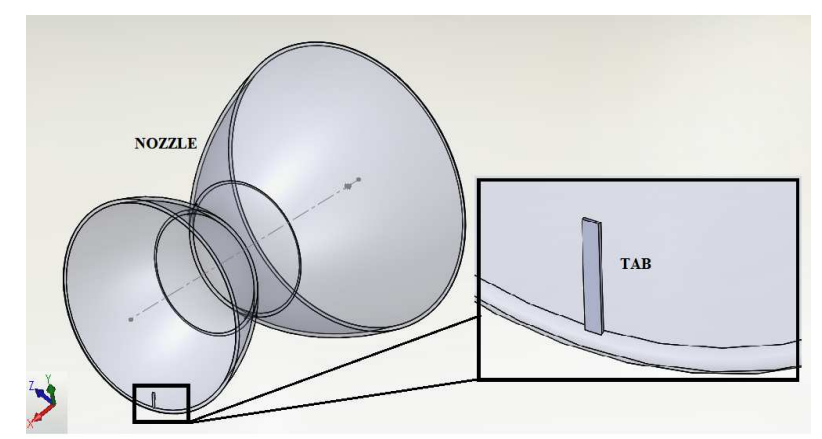

Figure (a.): using tab in Nozzle circumference as passive control of Jet. 


\section{LITERATURE SURVEY}

Winant \& Browand [6] using Experimental method in mixing of two streams of water in different velocity by controlling the growth of mixing layers in the Lucite-walled channel. The result of this develops vortices-carry fluid in intersections. The shear layer is usually in a turbulent state. Main output studies are that the mixing layer that consists of the large-scale vertical structure with axes perpendicular to the direction of the mean flow. This structure derives energy directly from the velocity being rolled along by the difference in velocity across the mixing layer. The turbulent mixing layer grows is called 'Vortex paring'.

S C M Yu, Y X Hou and S C Low [7] analyzed flow characteristics of a square jet with a mixing tab using a two-component laser Doppler anemometer at Reynolds number of 1.026 X 105. In comparing to the rectangular tab to the triangular tab, a rectangular tab gives $35 \%$ more growth of streamwise and decays faster with downstream distance. To reduce noise and mixing encasement investigation done by E. Rathakrishnan \& S.B.verma [8] by the experimental method with the different angle of sharp notches geometry. This examination is taken out for (I) correctly extended jets, with the sonic speed at the opening way out and (ii) Underexpanded sonic planes with under expansion level 2.0. Compare Plots of Mac No. incorrectly and Underexpanded condition $(\mathrm{Mj}=1.58)$ with a Square notch and Triangular notch, Square notch is expected to have a better plot relative to others. Pankaj Kr. Singh \& E. Rathakrisnan [9] established experimentally that tab produces the pairs of counter-rotating vortices, whereas the Half delta wing vortex produces only a simple vortex. The $80 \%$ reduction was in central line decay found when the comparison between tabbed and untapped nozzle is taken for Underexpanded sonic jet using two rectangular tabs at the Nozzle exit with compare to correctly expanded jet. L.P. Chua , S.C.M. Yu, X.K. Wang[10] has Investigated experimentally that using square jet and four triangular shape small tabs downstream $\theta=450$ for strong “fingers", are formed. At $\theta=1350$, a construction of four weak "fingers" plus four " "mushrooms" between two adjoining fingers is formed. The normalized cross-section dispersion area of the three jets investigated shows that the square jet with the downstream tilted tabs has the major ratio at $\mathrm{x} / \mathrm{D}>1.2$. The research aimed was to optimize between the increase of mixing and the reduction of the thrust of the jet with and without tab. By the experimental method, Shantanu Srivastava and E. Rathakrishnan [11] evaluated the efficiency of limiting tab to reducing the core length and improving the mixing and promoting the small scale vortices. The analysis was done in the presence of subsidiary adverse pressure gradient, almost zero pressure gradients, and insignificant approving pressure gradients at Mach 1.86 square nozzle exit. It is observed that the presence of a tab improves the early mixing of the supersonic jet. The centreline pressure decay plot for uncontrolled and controlled jets it is shown that the tab in the form of cross-wire is capable of enhancing jet mixing as the jet comes out of the nozzle. Another advantage comes out by using the tab in the form of cross wire is that subsonic zone is developed near the cross wire due to shock wave. This subsonic zone gives more time of interaction, as a result of improvement in mixing. The core length reduction for NPRs 5, 5.5, and 6 are $87.5 \%, 87.5 \%$, and 77\% for respectively and maximum reduction comes out to be $87.5 \%$.

\section{NUMERICAL MODELS}

The ANSYS FLUENT used to solve the principal governing equations for mass, momentum, and energy. These conditions, inconsistent state conditions, are illuminated with twofold exactness precision, a way to deal with relieving the impacts of average adjust errors. A second-order upwind scheme is used for modeling the flow, the turbulent kinetic energy, and other convective variables. The principal governing equations for fluid flow are the accompanying. The overseeing conditions 
comprise of the progression condition and the Reynolds-found the middle value of administering conditions for consistent compressible violent stream combined with the condition of the state, $p=\rho R T$. The arrangement of the overseeing conditions can be depicted as follows:

\section{The Continuity Equation}

"The rate of change of mass of a fluid element is equal to the net rate of mass flow across its control volume". The two-dimensional continuity equation for compressible flow of jet can be expressed [12] as;

$$
\frac{\partial}{\partial x_{i}}\left(\rho u_{i}\right)=0
$$

$\mathrm{u}$ is component of velocity along $\mathrm{x}$ and $\rho=\rho(\mathrm{x})$ is the density of air.

(i)

\section{RANS Equation}

$\frac{\partial}{\partial x_{j}}\left(\rho u_{i} u_{j}\right)=-\frac{\partial p}{\partial x_{i}}+\frac{\partial}{\partial x_{j}}\left[\mu\left(\frac{\partial u_{i}}{\partial x_{j}}+\frac{\partial u_{j}}{\partial x_{i}}-\frac{2}{3} \delta_{i j} \frac{\partial u_{l}}{\partial x_{l}}\right)\right]+\frac{\partial}{\partial x_{j}}\left(-\rho \overline{u_{i}^{\prime} u_{j}^{\prime}}\right)$

Where $u$ denotes mean quantities and the $\mathrm{u}^{`}$ fluctuating or turbulence quantities, $\mathrm{p}$ is pressure, $\boldsymbol{\rho}$ is density, $\mu$ is viscosity. The additional fluctuating $\operatorname{mtantities~}_{i} n_{j}$ are the unknowns turbulent or Reynolds-stress tensors, while represents the velocity fluctuation in i-direction. These eqnations are not a closed set and turbulence is required to model the turbulent or Reynolds-stress tensors [14].

\section{(ii) Energy Equation}

The energy equation is the law of conservation of energy, which states that the rate of change of energy inside the fluid element is equal to the sum of net flux of heat into the element and the rate of work done on the element due to body and surface forces. The energy equation for steady compressible flow can be mathematically expressed as [13];

$$
\frac{\partial}{\partial t}(\rho E)+\frac{\partial}{\partial x_{i}}\left[u_{i}(\rho E+p)\right]=\frac{\partial}{\partial x_{j}}\left(\left(k+\frac{C_{p} \mu_{t}}{0.85}\right) \frac{\partial T}{\partial x_{j}}+u_{i}\left(-\rho \overline{u_{i}^{\prime} u_{j}^{\prime}}\right)\right)
$$

\section{Turbulence Models}

The turbulence model is a computational methodology to close the arrangement of mean stream conditions. For most engineering applications it is superfluous to determine the subtleties of the violent changes. We just need to know how disturbance influenced the mean stream. Specifically, we need articulations for the Reynolds stresses.

\section{The k-equation}

The equation for k contains additional turbulent fluctuation terms that are unknown. Again using the Boussinesq assumption, these fluctuation terms can be linked to the mean flow. The following (simplified) model equation for $\mathrm{k}$ is commonly used[15].

$$
\frac{\partial}{\partial x_{j}}\left(\rho u_{j} k\right)=\frac{\partial}{\partial x_{j}}\left[\left(\mu+\frac{\mu_{t}}{\sigma_{k}}\right) \frac{\partial k}{\partial x_{j}}\right]+\rho\left(P_{k}^{*}-\beta_{1} \varepsilon-\beta_{2} k \omega\right)
$$

The Prandtl number $\sigma_{\mathrm{k}}$ connects the diffusivity of $\mathrm{k}$ to the eddy viscosity. Typically a value of 1.0 is used. 


\section{The $\varepsilon$-Equation}

A model equation for $\varepsilon$ is derived by multiplying the $\mathrm{k}$ equation by $(\varepsilon / \mathrm{k})$ and introducing model constants. The following (simplified) model equation for $\varepsilon$ is commonly used.

$$
\frac{\partial}{\partial x_{j}}\left(\rho u_{j} \varepsilon\right)=\frac{\partial}{\partial x_{j}}\left[\left(\mu+\frac{\mu_{t}}{\sigma_{\varepsilon}}\right) \frac{\partial \varepsilon}{\partial x_{j}}\right]+\frac{\rho}{T}\left(C_{1 \varepsilon} P_{k}-C_{2 \varepsilon} \varepsilon+C_{3 \varepsilon} \frac{P_{k}^{2}}{\varepsilon}\right)
$$

The Prandtl number $\sigma_{\varepsilon}$ connects the diffusivity of $\varepsilon$ to the eddy viscosity. Typically a value of 1.30 is used. Typically values for the model constants $\mathrm{C} 1 \varepsilon$ and $\mathrm{C} 2 \varepsilon$ of 1.44 and 1.92 are used [16].

\section{THEORETICAL MODEL}

The axisymmetric nozzle convergent Divergent model is shown in the figure. This C-D nozzle is designed to analyzed the mixing performance for Mac no 1.86 with a diameter of $12.28 \mathrm{~mm}$ with a nozzle throat diameter of $10 \mathrm{~mm}$. The dimension is designed for the formula (1.).

$$
\left(\frac{A}{A_{\mathrm{t}}}\right)^{2}=\frac{1}{M^{2}}\left\{\frac{2}{\gamma+1}\left[1+\frac{1}{2}(\gamma-1) M^{2}\right]\right\}^{\frac{\gamma+1}{\gamma-1}}
$$

The axisymmetric nozzle model length 30D and length of transfer direction is $15 \mathrm{D}$ about the jet axis

\section{Boundary Condition}

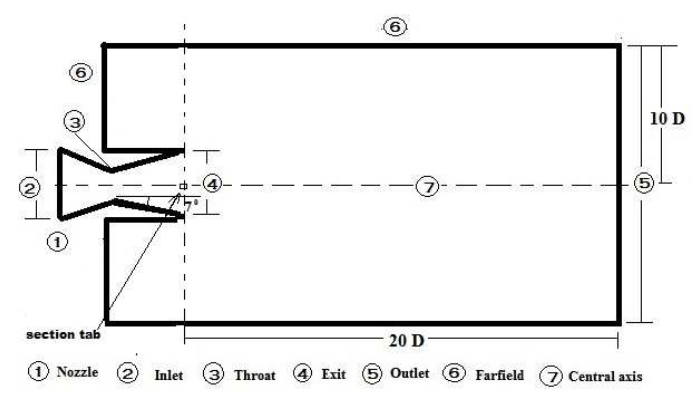

Figure (b): Computational Domain.

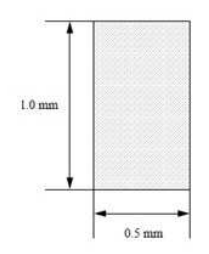

\section{Figure (c): Cross-section of Limiting Tab is}

used in the model.

Figure (b) shows the nozzle Inlet (2) At the nozzle inlet boundary pressure inlet is defined for NPR 4 and at a constant temperature, $300 \mathrm{~K}$. Nozzle outlet (5) at the nozzle outlet is defined. Pressure Far-field (6) in the flow domain the pressure 
far-field boundary condition is specified for boundary condition. The wall boundary (3) condition is specified for nozzle walls and assumes the no-slip condition. The axis of jet represents by (7.).The specification of the limiting tab is $1.0 \mathrm{~mm} \times 0.5 \mathrm{~mm}$ that is used in the nozzle for enhancing the mixing figure (c. ) The boundary conditions described for flow through the density-based turbulent C-D nozzle model in the process are given below: -

\begin{tabular}{|c|c|}
\hline \multicolumn{2}{|c|}{ At the inlet of the nozzle } \\
\hline Pressure $(\mathrm{Pi})$ & $=4 * 101325 \mathrm{pi}$ \\
\hline $\mathrm{T}$ & $=300^{\circ} \mathrm{K}$ \\
\hline At the nozzle surface, $\mathrm{Vx} 1$ & $=0$ \\
\hline Fluid & $=$ air (ideal gas) \\
\hline Ideal gas viscosity & $=$ Sutherland \\
\hline
\end{tabular}

\section{Mesh Generation}

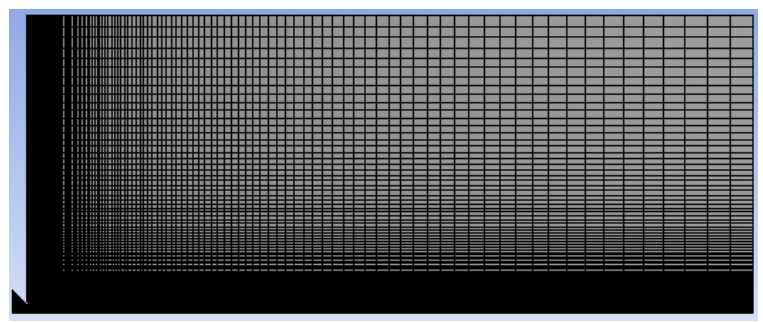

Figure (d): Computational grid for controlled jet.

The grid independence test, carried for 2-D quadrilateral Figure (d) structured grids sizes of 56016, 87237, 114274, 126036, 154680, 223581, and 349117. The Mach number of the jet issuing from the nozzle is computed at the different spatial locations along the jet centerline, downstream of the nozzle exit. The computation of the Mach number at different grid size results Figure (e) in finding the grid for which the solution becomes independent of the grid size. The results are found to be independent of the grid for the minimum grid size of 223581 at all spatial locations, downstream of the nozzle exit, along the jet centerline. Thus, the grid of 223581 is adopted for the complete simulation of the mixing characteristics of the uncontrolled jet. The maximum skewness of 0.43 and a maximum aspect ratio of 2.38 are reported for the present grid size of 223581 .

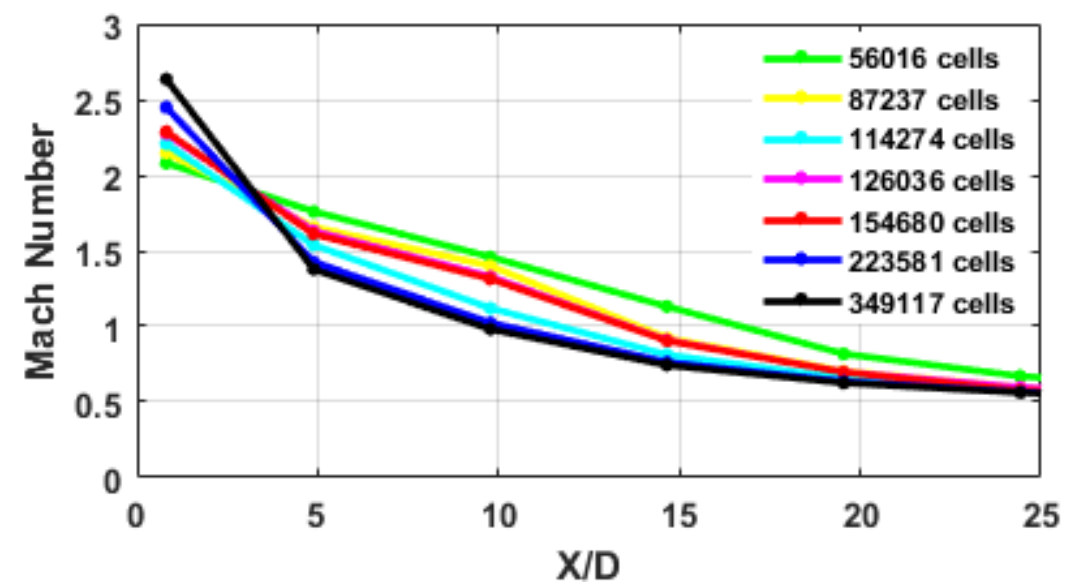

Figure (e): Grid Independence test for uncontrolled jet.

\section{RESULTS}

Mach number is defined as the ratio of the local velocity of the flow to the local sonic velocity. Mach number is greater than 
unity for subsonic flows and less than it for supersonic flows. The flow is called sonic when the Mach number is equal to one. A figure 11 shows the comparison of variation of Mach number plot for different mathematical models for downstream of the nozzle exit along the jet centerline. The Mach number variation of the jet from the Mach 1.86 convergent-divergent nozzle of the circular cross-section from inlet to exit, at NPR 4 is shown in Figure (f). At this NPR, the jet is overexpanded with an overexpansion level of about $36.4 \%$ of SST $\mathrm{k} \omega$ and Standard $\mathrm{k} \omega$. As the jet further moves downstream of the nozzle, there is an increase in the Mach number which indicates the gain in the jet momentum due to the expansion fans, as a result of unlike reflection from the free boundary. The jet propagating downstream of the nozzle exit meets the oblique shocks and expansion fans alternately, as a result of unlike reflection from the free boundary of the jet. Furthermore, it is seen that the Mach number progressively reduces downstream of the nozzle exit which might be due to the jet mixing.

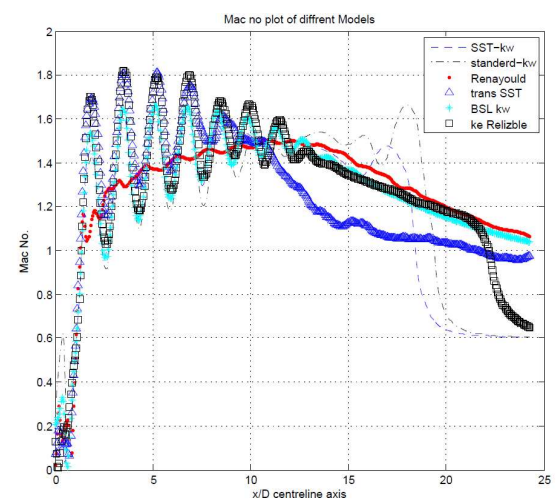

Figure (f): Mac Number plot of different Turbulent model.

The jet mixing causes the viscous activity to reach the jet centerline from the jet boundary as the jet moves downstream of the nozzle exit. Figure (g) It is interesting to see the continuous reduction in the Mach number beyond $\mathrm{x} / \mathrm{D}=16$ which indicates the nearing end of the jet core. Beyond $x / D=20$, the jet centerline velocity becomes subsonic, indicating the end of the jet core and the beginning of the characteristic decay zone. Beyond $\mathrm{X} / \mathrm{D}=18$, the jet attains a self-similar profile with Mach number less than 0.6. Comparing to core lengths of all mathematical models due to the tab in controlled jet mixing is started earlier and oblique shocks are produced. It is model capabilities that capture the clear shocks by numbers of peak variations along the core length In comparing all mathematical models k-e Relizble shows fully develop by some peaks of oblique shock and approaching developing zone in core length. Transition SST also captured the fully developed turbulent flow and shock by mixing in potential core

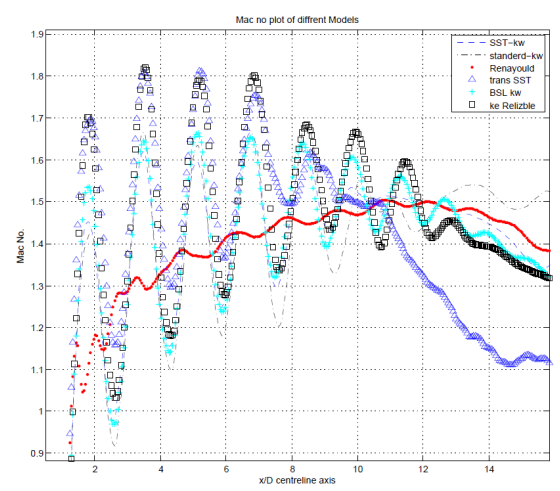

Figure (g): Mac Number plot shows Oblique shock peaks of different turbulent models of different Turbulent model. 


\section{CONCLUSIONS}

The present work shows the comparison of simulation plots of different turbulent models of Mac No = 1.86 and NPR 4 in presences of rectangular cross-section tab in the exit of CD nozzle of step size 1. Total CPU time taken for one set of iteration for $i 7$ processor was 5 hrs. The results are presents as Mac No non-dimensional plots of different turbulent models on CPD (centre pressure decay)

(a) Six turbulent models (SST k $\omega$, Standard k $\omega$, Reynolds, transition SST, BSL k $\omega$, k e realizable) are investigated in the presence of passive jet controls.

(b) The centerline pressure decay plots obtain from the simulation. k e realizable and transition SST captured all the maximum number of shock cells in the potential core region.

(c) The number of shock cells and core length is covered by k e realizable and transition SST can reach the fully developed region.

(d) For further study k e realizable and transition SST turbulent models can be used.

\section{REFERENCES}

1. Bassi, Francesco, et al. "Discontinuous Galerkin solution of the Reynolds-averaged Navier-Stokes and k- $\omega$ turbulence model equations." Computers \& Fluids 34.4-5 (2005): 507-540.

2. Girimaji, Sharath S. "Partially-averaged Navier-Stokes model for turbulence: A Reynolds-averaged Navier-Stokes to direct numerical simulation bridging method." (2006): 413-421.

3. Mayer, John P., Harold A. Hamer, and Carl R. Huss. "A study of the use of controls and the resulting airplane response during service training operations of four jet fighter airplanes." (1954).

4. Nastase, Ilinca, and Amina Meslem. "Passive control of jet flows using lobed nozzle geometries." Mechanics \& Industry 8.2 (2007): 101-109.

5. Sadeghi, H., and A. Pollard. "Effects of passive control rings positioned in the shear layer and potential core of a turbulent round jet." Physics of fluids 24.11 (2012): 115103.

6. Winant, Clinton D., and Fred K. Browand. "Vortex pairing: the mechanism of turbulent mixing-layer growth at moderate Reynolds number." Journal of Fluid Mechanics 63.2 (1974): 237-255.

7. Yu, S. C. M., Y. X. Hou, and S. C. Low. "The flow characteristics of a confined square jet with mixing tabs." Proceedings of the Institution of Mechanical Engineers, Part G: Journal of Aerospace Engineering 212.2 (1998): 63-76.

8. Verma, S. B., and E. Rathakrishnan. "An experimental study on the noise characteristics of notched circular-slot jets." Journal of sound and vibration 226.2 (1999): 383-396.

9. Kaushik, Mrinal, Pankaj Singh Thakur, and E. Rathakrishnan. "Studies on the effect of notches on circular sonic jet mixing." Journal of propulsion and power 22.1 (2006): 211-214.

10. Wang, X. K., L. P. Chua, and S. C. M. Yu. "On the near-field of a square jet with vortex-generating tabs." Fluid Dynamics Research 32.3 (2003): 99.

11. Rathakrishnan, E., and Shantanu Srivastava. "Performance of Corrugated Limiting Tab in Presence of Sharp Corners." 49th AIAA/ASME/SAE/ASEE Joint PropulsionConference. 2013. 3975. 
12. Rathakrishan E., Applied Gas Dynamics, $2^{\text {nd }}$ ed., Wiley India Pvt. Ltd., 2010.

13. Anderson, J., Degrez, G., Degroote, J., Dick, E., Grundmann, R. and Virendeels, J. Computational Fluid Dynamics: An Introduction. Springer Verlag and Academic Press, 3 rd edition, (2009), Berlin, Heidelberg.

14. Swanson, R. C., and C-C. Rossow. "An efficient solver for the RANS equations and a one-equation turbulence model." Computers \& Fluids 42.1 (2011): 13-25.

15. Chai, Xiaochuan, and Krishnan Mahesh. "Dynamic-equation model for large-eddy simulation of compressible flows." Journal of Fluid Mechanics 699 (2012): 385-413.

16. Xu, Jiangrong, et al. "Algebraic stress model with RNG E-equation for simulating confined strongly swirling turbulent flows." Journal of Thermal Science 10.1 (2001): 14-19. 

